\title{
RETOS PARA EL DERECHO DEL TRABAJO EN CUBA FRENTE A LA REGULACIÓN DE LAS TÉCNICAS DE REPRODUCCIÓN HUMANA ASISTIDA
}

\author{
Reynaldo Jorge Lam Peña ${ }^{1}$
}

Roxana Blanco Martínez ${ }^{2}$

\section{Resumen}

El presente artículo de investigación científica pretende analizar las técnicas de reproducción humana asistida (TRHA) como derechos sexuales y reproductivos y su repercusión en el ámbito jurídico laboral cubano a partir los futuros retos que se le presentan a la normativa cubana vigente sobre la base de los cambios sociales actuales vinculados a la importancia de una maternidad y paternidad responsable; debido a que existen en Cuba imperfecciones jurídicas en cuanto a la protección de las TRHA, pues la norma que protege la maternidad no se adecua a los nuevos avances científicos y concepciones modernas sobre las familias. La investigación se elaboró a partir de un estudio de documentos teóricos junto a métodos científicos como el exegético normativo, que busca el análisis de las normas jurídicas; el jurídico- doctrinal que combina los aspectos teóricos necesarios para entender las categorías que se ponen en discusión en este material y el jurídico comparado para analizar el fenómeno en el contexto latinoamericano. De esta forma se analizó el concepto y contenido de las TRHA y la necesidad de su repercusión en el marco laboral para luego exponer la trascendencia al ámbito laboral y familiar.

Palabras claves: derechos reproductivos y sexuales; técnicas de reproducción asistida; derechos en el trabajo;

\section{Resumo}

Este artigo de pesquisa científica tem como objetivo analisar as técnicas de reprodução humana assistida (TRHA) como direitos sexuais e reprodutivos e sua repercussão no campo jurídico trabalhista cubano com base nos desafios futuros que são apresentados aos regulamentos cubanos atuais com base nas mudanças sociais atuais relacionadas a a importância da maternidade e paternidade responsáveis; Porque existem imperfeições legais em Cuba em relação à proteção do TRHA, uma vez que a norma que protege a maternidade não se adapta a novos avanços científicos e concepções modernas de famílias. A pesquisa foi realizada a partir de um estudo de documentos teóricos, juntamente com métodos científicos como o exegético normativo, que busca a análise de normas legais; a doutrina jurídico que combina os aspectos teóricos necessários para entender as categorias discutidas neste material e a jurídica comparada para analisar o fenômeno no contexto latino-americano. Dessa forma, analisaram-se o conceito e o conteúdo do TRHA e a necessidade de sua repercussão no ambiente de trabalho, sendo então exposta a importância do trabalho e da família.

Palavras-chave: direitos reprodutivos e sexuais; técnicas de reprodução assistida; direitos no trabalho

\footnotetext{
${ }^{1}$ Reynaldo Jorge Lam Peña. Profesor de Derecho del Trabajo y de la Seguridad Social, Facultad de Derecho, Universidad de La Habana, Cuba. Juez Profesional Suplente no Permanente del Tribunal Provincial Popular de la Habana, Cuba. Maestrante en Derecho Constitucional y Administrativo por la Universidad de La Habana, Cuba. ORCID: 0000-0003-0629-4612. Contacto: reynaldo@lex.uh.cu

2 Roxana Blanco Martínez. Estudiante de 5to año de la Licenciatura en Derecho. Facultad de Derecho, Universidad de La Habana, Cuba. Alumna Ayudante de Derecho del Trabajo y de la Seguridad Social en el Departamento de Asesoría Jurídica e Internacional de la Universidad de La Habana. Contacto: roxana@lex.uh.cu
} 


\section{INTRODUCCIÓN}

El Derecho como ciencia no es inmutable, sino que debe adecuarse a las cambiantes situaciones de la realidad social y del vertiginoso avance científico. Ello debe efectuarse sobre la base de los valores y principios que sustentan nuestro ordenamiento jurídico a fin de contar con una legislación remozada y actual; toda vez que pueden existir ciertos vacíos en las normas discordantes con nuestra realidad.

El desarrollo de nuevos procesos médicos y biotecnológicos para la reproducción humana no es la excepción a la regla y por ello resultan un objeto de análisis interesante para el Derecho. Las Técnicas de Reproducción Humana Asistida, en lo adelante TRHA, se definen por la Organización Mundial de la Salud (2010) como todos los tratamientos o procedimientos que incluyen la manipulación tanto de ovocitos como de espermatozoides o embriones humanos para el establecimiento de un embarazo. Las principales causas que provocan su empleo son los problemas de infertilidad o esterilidad. Independientemente de ello, hay otros factores que conducen al aumento en el acceso a dichos tratamientos a la hora de procrear: casos de parejas que desean reservar sus embriones para gestarlos más adelante (por ejemplo, tras la curación de un cáncer); mujeres sin parejas que desean tener un hijo en edad temprana postergando el matrimonio o una pareja estable; mujeres solteras que no desean casarse ni tener pareja estable; parejas homosexuales; parejas o mujeres solas que desean evitar el riesgo de ciertas enfermedades genéticas en su hijo; la generación de "bebés-medicamento" para tratar a otros; la obtención de células troncales para clonación o embriones para investigación, y un sinfín de posibilidades más. Incluso ya se recurre a ellas como primera alternativa sin intentar siquiera remediar la causa de esterilidad. (Gómez y Navarro, 2017, p. 3)

Surge para el ordenamiento jurídico el deber de regular las implicaciones jurídicas de estas técnicas por una cuestión de justicia social y de garantía a los derechos reproductivos y sexuales. Para el Derecho laboral, resulta evidente que la situación de los trabajadores y, sobre todo, las trabajadoras que utilizan estas técnicas impactará de lleno en la dinámica de su relación laboral. En efecto, tales tratamientos generan al trabajador una serie de necesidades específicas relacionadas que exigirá una protección normativa al respecto.

En Cuba de la lectura de la nueva Constitución de la República (2019), sobresale el desarrollo de una gama de derechos a tono con las organizaciones y los instrumentos internacionales de los que forma 
parte y ha ratificado 3 . Se reconoce la dignidad humana como derecho y como valor supremo (Artículo 40); el aseguramiento por parte del Estado de los derechos sexuales y reproductivos de la mujer (Artículo 43), el derecho al trabajo (Artículo 64) y otros derechos en el trabajo (Artículo 65 al 69); el derecho a fundar familia (Artículo 81) y la protección por parte del Estado de la maternidad y la paternidad (Artículo 84); los cuales deben analizarse de manera armónica, en tanto su interpretación y aplicación le imponen nuevos retos al Derecho del Trabajo, para garantizar su pleno disfrute.

En el país resulta necesario la aplicación de estas técnicas (Gran y López, 2003), en gran parte, por la declinación de la capacidad reproductiva (infertilidad) de la población ante la morbilidad por infecciones de transmisión sexual, envejecimiento poblacional, razones que producen conductas que inciden en la procreación como la disminución de la mortalidad infantil, preescolar y escolar, la cual garantiza y otorga seguridad a la pareja en cuanto a la sobrevivencia de los hijos tenidos. También influyen componentes socioeconómicos, como la incorporación de la mujer a la actividad educacional y laboral; cultura social en general y sanitaria, en particular de la población en la buena práctica de la maternidad segura, oportuna y responsable, así como de la crianza de los hijos; la aún presente dificultad de vivienda para las parejas, como un elemento de decisión importante para la reproducción; el nivel educacional y profesional de las personas en nuestro país.

La práctica de la interrupción de un embarazo no deseado de una manera legal e institucional y en condiciones de seguridad y las acciones desarrolladas en el campo de la planificación familiar, en función de preservar y respetar la salud y los derechos reproductivos de la mujer y de la pareja, han tenido un importante impacto en el descenso de la fecundidad, y es un componente de la anticoncepción.

Además, la población "fecundable" ha decrecido en los últimos diez años y la existente, en numerosas ocasiones ha alcanzado ya el tamaño deseado de la familia, el que según los estudios recientes siguen expectativas de pocos hijos o familias pequeñas por mutuo acuerdo de la pareja.

\footnotetext{
${ }^{3}$ Dentro de ellos se incluyen los Convenios 3, 103 y 183 de la Organización Internacional del Trabajo (OIT), de los años 1919 , 1952 y 2000 respectivamente. También está la Recomendación 191/2000. Asimismo se encuentra la convención para la Eliminación de todas formas de Discriminación contra la Mujer; la Conferencia Internacional sobre la Población y el Desarrollo de El Cairo de 1994; la Declaración y la Plataforma de Beijing de 1995; la Declaración Universal sobre el Genoma Humano y los Derechos Humanos; la Declaración de Bioética de Gijón; la Declaración Universal sobre Bioética y Derechos Humanos, entre otros.
} 
Con el objetivo de contribuir al incremento de la natalidad en el país, y dando respuesta al Lineamiento de la Política Económica y Social del Partido y la Revolución (2016) que atañe al contexto demográfico, el Ministerio de Salud Pública desarrolla el Programa de Atención a la Pareja Infértil. Las parejas con dificultades para concebir un embarazo pueden intentar la gestación, asistidas por profesionales altamente cualificados y con la garantía de un equipamiento de primer nivel tecnológico; todo esto en consonancia con los principios básicos de nuestra medicina: humana y libre de costo.

El embarazo y la maternidad son épocas de particular vulnerabilidad para las trabajadoras y sus familias y por ello requiere de una especial protección para salvaguardar su salud y la de sus hijos. Estas necesitan un tiempo adecuado para dar a luz, para su recuperación y para la lactancia. Al mismo tiempo, requieren protección laboral no sólo en cuanto a la igualdad en el acceso al empleo, sino que también les garantiza el mantenimiento de unos ingresos (salario) que a menudo son vitales para el bienestar de toda su familia. Sin embargo, si bien en las instituciones y los centros laborales cubanos, se promueve y apoya a las trabajadoras gestantes, no existe una regulación legal que respalde o proteja al trabajador durante el tiempo que se somete a estas prácticas de reproducción artificial.

La situación anterior constituye una evidencia de lagunas o vacíos legislativos, pues es importante resaltar que durante la realización de estos procedimientos para lograr la concepción, la mujer trabajadora no se encuentra todavía embarazada, de ahí que no se encuentre en un período pre ni postnatal, establecidos en Decreto Ley 339 "De la Maternidad de la Trabajadora" y por ende pudiera resultar dudoso si le asisten derechos al igual que a la madre y al padre trabajadores. ${ }^{4}$

La problemática del empleo de las técnicas de reproducción asistida y su repercusión en el ámbito laboral de la mujer trabajadora y el ejercicio pleno de sus derechos ha desbordado el ámbito de lo estrictamente personal, familiar y médico, y se está configurando como objeto que reclama una intervención; de ahí que le concierne a organismos e instituciones sociales, al igual que al Estado,

${ }^{4}$ Vid. Artículos 1 y 2 del Decreto Ley 339/2017 "De la Maternidad de la Trabajadora" (GO Extraordinaria No.7 10/2/2017) Artículo 1: El objetivo de este Decreto Ley es conceder derechos a la madre y al padre trabajadores del sector estatal, en lo que a cada cual corresponda, para propiciar la responsabilidad compartida con la familia en el cuidado y atención del hijo e hija menores de edad, en lo adelante menor. A estos fines:

a) Asegura y facilita a la mujer trabajadora la atención médica durante el embarazo, el descanso pre y posnatal, la lactancia materna y, a ambos padres, el cuidado del menor;

b) en caso de fallecimiento de la madre, establece una protección al padre trabajador u otro familiar trabajador de los determinados en el presente, a quien se encargue el cuidado del menor; $y$

c) dispone un tratamiento diferenciado cuando el menor requiera de atenciones especiales.

Artículo 2: Los derechos contenidos en este Decreto Ley se originan por la condición de trabajadora de la madre. 
ofrecer soluciones de apoyo no solo por medio de políticas públicas sino también a través de mecanismos legales y jurídicos.

Por ello la presente investigación tiene como objetivo principal: analizar desde la regulación jurídica los elementos necesarios para garantizar la plena utilización de los trabajadores cubanos de las técnicas de reproducción asistida. Sobre la base del mismo se pretende explicar los aspectos teóricodoctrinales con respecto a las técnicas de reproducción humana asistida y los derechos sexuales y reproductivos; comparar la regulación internacional con respecto a la utilización de las técnicas de reproducción humana asistida; analizar las principales deficiencias en cuanto a la plena utilización de las Técnicas de Reproducción Humana Asistida del ordenamiento jurídico laboral cubano; y proponer los principales elementos que garanticen la plena utilización de las Técnicas de Reproducción Humana Asistida trabajadores cubanos.

Resulta de enorme importancia esta investigación ya que ofrece un cambio de visión jurídico-laboral en cuanto a la maternidad y la paternidad. Se evidencia fehacientemente la necesidad de proteger a los trabajadores, que se encuentran utilizando alguna de las TRHA, en la etapa de preconcepción pues ante el ordenamiento jurídico cubano actual se materializa un estado de indefensión en este sentido al carecer de reconocimiento. Además, hemos podido colegir que la utilización de estos procedimientos ya no se sustenta en problemas reproductivos o físicos sino en la autonomía de la voluntad y que constituye un mecanismo para fundar nuevas formas de familias.

\section{ACERCA DE LAS TÉCNICAS DE REPRODUCCIÓN HUMANA ASISTIDA}

La aplicación de las Técnicas de Reproducción Humana Asistida requiere de la colaboración estrecha de profesionales con formación clínica (ginecólogos, urólogos, andrólogos), especialistas en técnicas de laboratorio y de todo el personal de apoyo como psicólogos y personal de enfermería. Surgen con el objetivo de viabilizar la reproducción humana y permitir el acceso a la maternidad y a la paternidad a personas con problemas de esterilidad ${ }^{5}$ e infertilidad ${ }^{6}$, o que simplemente en virtud de la autonomía

\footnotetext{
${ }^{5}$ La esterilidad es la imposibilidad de lograr la fecundación y el embarazo. También se denomina esterilidad absoluta. La Federación Internacional de Ginecología y Obstetricia (FIGO), la Sociedad Europea de Embriología y Reproducción Humana (ESHRE) y la Organización Mundial de la Salud (OMS) no considera estéril a la pareja hasta que no hayan transcurrido, sin éxito, 2 años de relaciones sexuales regulares con finalidad reproductora. Vid Álvarez Plaza, Consuelo y Selmouni, Farida: "Reproducción humana asistida", disponible en https://www.academia.edu, consultado el 15/10/2019. ${ }^{6}$ La infertilidad hace referencia a que, si bien hay fecundación y gestación, los fetos no logran la viabilidad. Es sinónimo de esterilidad relativa. Según la Organización Mundial de la Salud (OMS), la infertilidad es "una enfermedad del aparato reproductor definida por la imposibilidad de lograr un embarazo clínico después de 12 meses o más de relaciones sexuales
} 
de voluntad deciden tomar la decisión de usarlas. Existen una variedad de procedimientos y su aplicación depende de las características de cada persona ${ }^{7}$.

La inseminación artificial (IA) consiste en el depósito de forma no natural de espermatozoides en el tracto reproductor de la mujer con el objetivo de conseguir una gestación. Puede ser: IA con semen conyugal, de pareja u homólogo o IA con semen de donante o heteróloga. La modalidad más aceptada es la homóloga ya que resulta menos conflictiva, pues ante la inseminación artificial heteróloga se generan contradicciones con el donante del material genético, con respecto a la filiación legal de la descendencia obtenida, así como el costo que representa mantener los bancos de esperma. Durante el proceso se requerirá de ecografías periódicas, consultas médicas reiteradas, análisis de sangre, etcétera.

La Fecundación o Fertilización in vitro que consiste en poner en contacto los gametos masculinos (espermatozoides) y femeninos (ovocitos) en pos de la fecundación y el desarrollo embrionario inicial fuera del organismo de la mujer, de aquí la diferencia con la inseminación artificial.

Asimismo, contamos con el Diagnóstico genético preimplantacional (DGP) que es un método de diagnóstico prenatal que permite seleccionar embriones sanos para su transferencia al útero, la elección del sexo y la selección inmunológica del embrión. Esta técnica puede resultar útil en personas afectadas o portadoras de enfermedades transmisibles genéticamente y debidas a la alteración de un gen; personas portadoras de alteraciones cromosómicas transmisibles y personas con mayor riesgo de alteraciones genéticas en sus gametos, que podrían determinar la formación de embriones genéticamente anormales.

La maternidad sustituta o maternidad subrogada o también conocida como alquiler de vientre ${ }^{8}$ consiste en la práctica en la cual una mujer, previo acuerdo entre partes, se compromete a llevar a cabo un embarazo y entregar al niño o niña fruto de ese proceso, en el momento del nacimiento o a los pocos días a otra persona o pareja renunciando a sus derechos de madre, con un fin altruista o a cambio de una suma de dinero. La complejidad de la práctica radica en las complicaciones jurídicas que genera: en cuanto a la determinación de la filiación materna que no se deriva del hecho biológico (el parto);

sin protección regular". Vid OMS: "Qué se considera infertilidad según la OMS, disponible en http://www.quechollodesegurodesalud.com, consultado el 15/10/2019

${ }^{7}$ Causa de esterilidad, calidad espermática, edad de la mujer, tiempo de duración de la esterilidad, etc

8 Vid ARÁmBULA REYES, A (2008) Maternidad subrogada. México: Centro de Documentación Información, Análisis, Cámara de Diputados, LX Legislatura. 
quién ejerce efectivamente la patria potestad del menor, etcétera. Incluso ello se agrava cuando se analizan las parejas homosexuales sobre todo masculinas para obtener descendencia. La relativa novedad de la técnica y sus implicaciones morales, sociales, legales han generado posiciones en contra y favor de esta.

Existen Tratamientos de Preservación de la Fertilidad que tienen como objetivo esencial conseguir un embarazo, pero no de forma inmediata sino aplazada en el tiempo. Esta decisión se puede tomar por diferentes circunstancias, por ejemplo: el diagnóstico de un cáncer. Los tratamientos quimioterapéuticos y radioterapéuticos a los que necesariamente deben ser sometidos para sobrevivir provocan, de manera irreversible, la pérdida de ovocitos y atrofia ovárica en las mujeres. Además, hay enfermedades no malignas que requieren tratamientos similares a los oncológicos (enfermedades autoinmunes). Quizás la mujer en virtud del libre desarrollo de su personalidad y de la voluntad procreacional no tiene un deseo de gestar inmediato y emplea las técnicas en aras de que la fecundidad asociada con la edad le impida ser madre. Algunos de estos tratamientos son: la congelación de ovocitos; la congelación de embriones; la congelación del tejido ovárico; la congelación del semen, etc.

\section{DERECHOS REPRODUCTIVOS Y SEXUALES}

La utilización de las TRHA presupone un derecho humano referido a la voluntad de tener descendencia biológica propia y se configura como una manifestación del libre desarrollo de la personalidad. Nos referimos al derecho a procrear, a los derechos reproductivos y sexuales. Dentro de los derechos sexuales podemos encontrar el derecho a decidir libremente sobre el propio cuerpo y sexualidad; derecho a decidir libremente con quién, cómo y cuándo ejercer la propia sexualidad; derecho a los servicios de salud sexual y derecho a la igualdad de oportunidades. Por otro lado, dentro de los derechos reproductivos se encuentra el derecho a decidir de forma libre y responsable el número y espaciamiento de los hijos e hijas; derecho a decidir libremente con quién, cuándo y cómo tener hijos e hijas; derecho a una educación oportuna e integral en salud reproductiva; derecho a acceder a servicios de aborto legal, seguro y de calidad y derecho a los servicios de salud reproductiva, libres de discriminación y de violencia. En esta segunda categoría hay dos aspectos importantes, establecidos tanto en el Sistema Universal como Interamericano para la Protección de los Derechos Humanos, la autonomía y la salud reproductiva que garantizan el acceso de las personas a técnicas y tratamientos de reproducción asistida. 
Las técnicas representan un factor de cambio social de apertura a múltiples posibilidades en el control de la reproducción que, va más allá de sus aspectos terapéuticos, pues constituye un medio alternativo de procreación, en función de la autonomía de la voluntad y un mecanismo para fundar nuevos modelos de familia (monoparentales u homoparentales). Se considera que el derecho a procrear es personal y que debemos respetar la decisión positiva o negativa de cada ser humano de convertirse en madre o padre y no sólo esto sino el método, la forma de ejercer ese derecho (ya sea de forma natural o asistida), así como la idea de usar métodos anticonceptivos y el aborto.

Además, la no discriminación es el corolario del principio de igualdad y contiene la posibilidad de permitir el acceso a TRHA no sólo a parejas heterosexuales sino también a parejas homosexuales e incluso a la mujer soltera que decide saciar sus deseos maternales y emprender ese camino sola. Además, la infertilidad al ser considerada como una discapacidad para procrear, negar el acceso a TRHA estaría atentando contra los postulados de la Convención Internacional de las Personas con Discapacidad (Valdez, 2017, p. 40)

Se hace necesario la existencia de disposiciones que respalden el proceso de concepción no natural como correlato de la salud reproductiva ya que si bien la infertilidad y la esterilidad no comprometen la existencia humana si puede traer consigo consecuencias psicológicas adversas para aquellos que integran la procreación en su proyecto de vida.

El Derecho Laboral debe insertarse en esta dinámica y en virtud del principio protectorio debe respaldar legalmente a aquellas mujeres que toman la decisión de ser madres. Existen normativas internacionales para proteger a la madre trabajadora, pero no hay referencia de las Técnicas de Reproducción Humana Asistida, pero es fundamental también extender la protección de los derechos laborales al tiempo que dure el proceso de concepción del embarazo para evitar situaciones de indefensión. Si tenemos en cuenta que durante el contexto de un tratamiento de este tipo la trabajadora podría verse en la necesidad de ausentarse de su puesto de trabajo de forma puntual para asistir a las consultas médicas pertinentes e incluso los padres comitentes de un contrato de gestación por sustitución también podría encontrarse en esa disyuntiva con motivo de la ejecución de trámites médicos y burocráticos; resulta necesario la regulación jurídica laboral que le brinde factibilidad al derecho a procrear y al derecho de fundar una familia y evitar así despidos injustificados, afectaciones económicas por reducción de la mensualidad del salario, etcétera. 


\section{CLABORJURI5

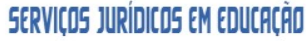

\section{PROTECCIÓN INTERNACIONAL DE LAS TRHA}

Una vez expuestas los principales cuestionamientos doctrinales en torno a las Técnicas de Reproducción Humana Asistida, consideramos pertinente realizar un estudio de la normativa jurídica internacional en pos de conocer el cómo se ha implementado su regulación en el mundo. De inicio cuestionaremos los principales instrumentos internacionales que representan una voluntad de hacer uniforme las cuestiones de reproducción artificial.

El reconocimiento de los derechos sexuales y reproductivos en el contexto internacional (Ver anexo l) ha tenido un desarrollo lento durante casi medio siglo. El primer referente en la materia lo encontramos en la Conferencia Mundial de Derechos Humanos de Teherán en 1968 donde es determinó que los padres tienen el derecho humano fundamental de determinar libremente el número de sus hijos y los intervalos entre los nacimientos. A pesar de que constituye un precedente fueron los movimientos feministas de los años 60 los que abrieron paso al surgimiento de estos derechos sobre la base de la reivindicación de los principios de igualdad y no discriminación y exigían que el Estado no interfiera en su libertad y debía garantizar las condiciones para ejercerla ${ }^{9}$

La Convención para la Eliminación de todas las formas de Discriminación contra la Mujer (1979) fue una batalla ganada por los movimientos feministas del siglo pasado en el ámbito jurídico, dicho tratado dio desarrollo a una de las principales premisas que formarán el corpus iuris para la protección de los derechos sexuales y reproductivos. Así, el artículo 16, literal e) de la Convención establece que los Estados tienen la obligación de asegurar tanto a hombres como mujeres "los mismos derechos a decidir libre y responsablemente el número de sus hijos y el intervalo entre los nacimientos y a tener acceso a la información, la educación y los medios que les permitan ejercer estos derechos". El anterior precepto desarrolla el derecho a fundar una familia, el cual había sido ya implementado en otros tratados y convenios de manera más general, como el Pacto Internacional de Derechos Civiles y Políticos, la Convención Americana de Derechos Humanos y el Convenio Europeo para la Protección de los Derechos Humanos y de las Libertades Fundamentales.

\footnotetext{
9 Vid COLECTIVO DE AUTORES: "El acceso a las técnicas de reproducción asistida como una gaantía de los Derechos Sexuales y Reproductivos: la jurisprudencia de la Corte Constitucional a la luz del Derecho Internacional de los Derechos Humanos", Revista Justicia No. 31, enero-junio, 2017, pg 175.
} 
En la Conferencia Internacional sobre la Población y el Desarrollo de El Cairo ${ }^{10}$ de 1994 , llevada a cabo en Egipto, se contó con la presencia de 179 representantes de países del mundo y se aprobó su Programa de Acción que vincula a todos los Estados participantes con carácter obligatorio.

En esta Conferencia se incluyó por primera vez la salud sexual y reproductiva como un verdadero compromiso frente al bienestar de las personas y la garantía de sus derechos. En dicha oportunidad se determinó que: La salud reproductiva es un estado general de bienestar físico, mental y social, y no de mera ausencia de enfermedades o dolencias, en todos los aspectos relacionados con el sistema reproductivo y sus funciones y procesos. En consecuencia, la salud reproductiva entraña la capacidad de disfrutar de una vida sexual satisfactoria y sin riesgos y de procrear, y la libertad para decidir hacerlo o no hacerlo, cuándo y con qué frecuencia. Esta última condición lleva implícito el derecho del hombre y la mujer a obtener información y de planificación de la familia de su elección, así como a otros métodos para la regulación de la fecundidad que no estén legalmente prohibidos, y acceso a métodos seguros, eficaces, asequibles y aceptables, el derecho a recibir servicios adecuados de atención de la salud que permitan los embarazos y los partos sin riesgos y den a las parejas las máximas posibilidades de tener hijos sanos.

Se realza el empoderamiento femenino y la aceptación de que toda persona tiene el derecho a decidir libre y responsablemente el número de hijos que quiere tener fue un triunfo frente a posiciones que afirmaban que debería ser la pareja quien decidiera de común acuerdo. Se debatió acerca del aborto y la disyuntiva de prescribirlo o despenalizarlo.

Su Programa de Acción en el Capítulo VII está referido a los derechos reproductivos y a la salud reproductiva y tiene como objetivos fundamentales asegurar el acceso a información amplia y fáctica y a una gama completa de servicios de salud reproductiva, incluida la planificación de la familia; propiciar y apoyar decisiones responsables y voluntarias sobre la procreación y sobre métodos que pueden elegirse para la regulación de la fecundidad que no estén legalmente prohibidos y asegurar el acceso a la información, la educación y los medios necesarios ${ }^{11}$. Considera que la atención a la salud reproductiva debería incluir tratamientos adecuados y prevención de la infecundidad, formas de

\footnotetext{
${ }^{10}$ Vid United Nations Population Information Network (POPIN). Informe de la Conferencia Internacional sobre la Población y el Desarrollo (El Cairo, 5 al 13 de septiembre de 1994), disponible en http://www.un.org/popin/icpd/conference/offspa/sconf13.html, consultado el 11 de octubre del 2019

${ }^{11}$ GALDOS SILVA, SUSANA: "La Conferencia en el Cairo y la afirmación de los derechos sexuales y reproductivos como base para la salud sexual y reproductiva", Revista Peruana de Medicina Experimental y Salud Pública No.30, 2013.
} 
interrupción del embarazo, prevención del aborto, servicios de atención prenatal, entre otras cuestiones.

La Cuarta Conferencia Mundial sobre la Mujer celebrada en Beijing, China, en septiembre de 1995 aprobó la Declaración y la Plataforma de Beijing de 1995. Dentro de sus disposiciones se reafirman los derechos femeninos como parte inalienable, integral e indivisible de los derechos humanos y libertades fundamentales; la potenciación del papel de la mujer en la sociedad; aboga por la igualdad de derechos, de oportunidades y de acceso a los recursos, la distribución equitativa entre hombres y mujeres de las responsabilidades respecto de la familia.

Se reconoce de modo explícito el derecho de las mujeres a controlar todos los aspectos de su salud, en particular su propia fecundidad.

En el año 2000, el Comité de Derechos Económicos, Sociales y Culturales, en su Observación General No. 14 sobre el derecho al disfrute del más alto nivel posible de salud, determinó que: "La mujer y el hombre están en libertad para decidir si desean reproducirse y en qué momento, y tienen el derecho de estar informados y tener acceso a métodos de planificación familiar seguros, eficaces, asequibles y aceptables de su elección, así como el derecho de acceso a los pertinentes servicios de atención de la salud"

En el año 2000 representantes de 189 países se congregaron en las Naciones Unidas en ocasión de la histórica Cumbre del Milenio y aprobaron un conjunto de objetivos que recogen aspectos fundamentales para la mejora de la salud reproductiva a nivel global: la reducción de la mortalidad materna y el avance hacia la igualdad entre los géneros unido al empoderamiento de las mujeres; mejorar la salud materna; combatir el VIH/SIDA y otras enfermedades; fomentar una alianza mundial para el desarrollo.

Estos estándares internacionales llevan a la conclusión que existe un vínculo irresistible entre estos dos aspectos, la salud y la autonomía reproductiva, por cuanto el no asegurar el libre ejercicio de esta última, necesariamente devendrá en una afectación a la primera. Esta consecuencia negativa en las libertades de las personas, a pesar de los esfuerzos por parte de la comunidad internacional para evitar su concreción, se evidencia con mayor ocurrencia, y especialmente en América Latina en casos de personas con infertilidad. 
Por esto el precedente más significativo que ha consolidado el surgimiento de las técnicas de reproducción asistida como garantías para el ejercicio de los derechos reproductivos, es el reconocimiento por parte de la Organización Mundial de la Salud (2009) de la Infertilidad como "una enfermedad del sistema reproductivo definida como la incapacidad de lograr un embarazo clínico después de 12 meses o más de relaciones sexuales no protegidas". Dicha posición es respaldada por diversos estudios que muestran cómo la infertilidad genera: “... severas consecuencias sociales, económicas y de salud en los habitantes de los países en desarrollo; los efectos físicos, emocionales y psicosociales negativos en la salud pueden no ser fácilmente detectables pero ya se han documentado $(\ldots)^{\prime \prime 12}$

La infertilidad es una condición individual y social, un individuo con infertilidad sufre de un cuerpo enfermo, una identidad humana sin cumplirse y presenta trastornos en sus relaciones con impacto social, político y económico.

Como respuesta a las necesidades de dicha población y con el fin de asegurar la garantía de los derechos sexuales y reproductivos de las personas, Estados como Argentina, y en menor medida Colombia recientemente, han venido reconociendo su obligación de brindar acceso a técnicas de reproducción asistida, mediante sus sistemas de salud a personas que de manera natural no pueden lograr dicho propósito. Estos avances en materia reproductiva se han materializado gracias al desarrollo que en términos de derechos humanos se ha venido gestando en el continente americano. Ahora existen tratados internacionales que en alguna medida tienen una mirada sobre los temas de Técnicas de Reproducción Humana Asistida. Según Burstein (2013) se puede encontrar:

\section{Convenio de Oviedo o Convenio relativo a los Derechos Humanos y la Biomedicina: Se aprobó por} el Consejo de Europa en 1996 y fue abierto a la firma en el año 1997. Plantea la prohibición de la clonación de seres humanos; la elección del sexo del ser humano que va a nacer, salvo que sea preciso para evitar una enfermedad hereditaria grave vinculada al sexo y la creación de embriones con fines de experimentación ${ }^{13}$. De igual forma permite la experimentación sobre embriones in vitro siempre que se garantice una protección adecuada del embrión y las intervenciones sobre el genoma humano cuando medien razones preventivas, diagnósticas o terapéuticas y sólo cuando no tenga por finalidad

\footnotetext{
12 Vid MCDONALD, E: A global perspective on infertility: An under recognized public health issue, University Center for International Studies, The University of North Carolina, 2004.

${ }^{13}$ Artículo 18 del Convenio de Oviedo
} 
la introducción de una modificación en el genoma de la descendencia ${ }^{14}$. Refiere que el cuerpo humano y sus partes no deben ser fuente de aprovechamiento ${ }^{15}$

Declaración Universal sobre el Genoma Humano y los Derechos Humanos: Aprobada por la ONU en 1997 reconoce al genoma humano como unidad fundamental de todos los seres humanos y patrimonio de la humanidad ${ }^{16}$. Estipula que cada individuo tiene el derecho a que se respete su dignidad con independencia de las características genéticas que posea y ello no puede ser un motivo para de discriminación pues se estaría atentando contra derechos humanos y libertades fundamentales ${ }^{17}$ Una investigación, un tratamiento o un diagnóstico relacionado con el genoma de un individuo debe contar con su consentimiento y ninguna de estas ni su aplicación al campo de la biología, la medicina o la genética puede prevalecer sobre los derechos humanos.

\section{Declaración de las Naciones Unidas sobre las Responsabilidades de las Generaciones Actuales} para con las Generaciones Futuras: Fue aprobada en la Conferencia General de la Organización de Naciones Unidas para la Educación, la Ciencia y la Cultura en su 29 reunión celebrada en París del 21 de octubre al 12 de noviembre del año 1997. Determina la responsabilidad de las generaciones actuales en cuanto al mantenimiento y perpetuación de la humanidad, respetando la dignidad humana. Dispone la necesidad de proteger el genoma humano y la diversidad biológica.

Declaración de Bioética de Gijón: fue aprobada en junio del 2000 y plantea que el genoma humano no puede ser patentable y constituye patrimonio de la humanidad. Concibe que una finalidad esencial de las Técnicas de Reproducción Humana Asistida es el tratamiento médico de los efectos de la esterilidad humana y facilitar la procreación si otras terapéuticas se han descartado por inadecuadas o ineficaces. Estas técnicas podrán utilizarse también para el diagnóstico y tratamiento de enfermedades de origen hereditario, así como en la investigación autorizada. Prohíbe la clonación humana y salvaguardar la dignidad humana en las prácticas investigativas y experimentales.

Declaración de las Naciones Unidas sobre la Clonación Humana, emitida el 8 de marzo del 2005, plantea que los Estados deben procurar y proteger la vida humana en la aplicación de las ciencias biológicas; prohibir cualquier modo de clonación humana y de aplicación de técnicas de ingeniería

${ }^{14}$ Artículo 13 del Convenio de Oviedo

${ }^{15}$ Artículo 21 del Convenio de Oviedo

${ }^{16}$ Artículo 1 de dicha Declaración

${ }^{17}$ Artículo 2 y 7 de la Declaración Universal sobre el Genoma Humano y los Derechos Humanos. 
genética que vayan en contra de la dignificación del ser humano y su protección. Asimismo, reguló la pertinencia de que los países tomen en cuenta la financiación de investigaciones médicas que tributen a las ciencias biológicas; a enfermedades como el VIH/SIDA, la tuberculosis y la malaria.

Declaración Universal sobre Bioética y Derechos Humanos: emitida el 19 de octubre del 2005 por la Organización de Naciones Unidad para la Educación, la Ciencia y la Cultura. Se reivindica el respeto a la dignidad humana, de los derechos y libertades fundamentales y protege los progresos científicos de los individuos de forma privilegiada. Con respecto a ello debemos disminuir los daños en la aplicación de los conocimientos científicos, prácticas médicas o tecnologías asociadas.

A partir de este recuento legislativo internacional podemos concluir que existe una tendencia encaminada a la protección del genoma humano como patrimonio humanitario para garantizar la vida y la supervivencia. Por ello resulta uniforme la idea de implementar medidas y normas que respalden de manera efectiva dicha defensa. Se estimulan las investigaciones científicas y médicas vinculadas a este bien jurídico, pero siempre respetando la dignidad humana. Asimismo, es semejante la prohibición de la clonación humana lo cual consideramos muy acertado para evitar la comercialización y el lucro con algo tan intrínseco al individuo. Además, se resguarda los derechos reproductivos y sexuales sobre la base del libre desarrollo de la personalidad en aras de garantizar el derecho a la salud de manera general.

Esta amplia gama de instrumentos internacionales fundamenta nuestra investigación ya que justifican la lucha de todos aquellos seres humanos que deciden reproducirse artificialmente y necesitan de legitimidad en su actuar. Además, requieren de la protección legal en cuanto a la filiación, los derechos sobre el niño o niña; los derechos laborales durante el proceso, etcétera. Los derechos reproductivos y sexuales requieren de garantías para su libre ejercicio y por medio de estos instrumentos internacionales se exterioriza la preocupación internacional en este sentido. No solo ello, sino que dichos instrumentos le ponen coto a la utilización de las TRHA pues actualmente la delincuencia organizada se lucra aprovechándose del deseo reproductivo de muchas personas y comercializa con el genoma humano lo cual está prohibido porque va en contra de la dignidad y de los derechos humanos.

\subsection{Convenios de la Organización Internacional del Trabajo (OIT)}

La Organización Internacional del Trabajo se ha preocupado a lo largo de la historia por preservar la salud de la madre y del recién nacido; habilitar a la mujer para que pueda combinar satisfactoriamente 
su rol reproductivo y su rol productivo; prevenir el trato desigual en el trabajo; y promover el principio de igualdad de oportunidades y de trato entre las mujeres y los hombres. Sin embargo, la todavía reciente aplicación de las técnicas de reproducción asistida hace que puedan darse situaciones que impidan que estas pretensiones puedan efectivamente llevarse a vías de hecho, afectándose los derechos en el trabajo de una trabajadora, inmersa en la utilización de estas técnicas para poder procrear. En correspondencia con ello, la OIT ha adoptado tres Convenios ${ }^{18}$ sobre la protección de la maternidad en diferentes años: en 1919, 1952 y, el más reciente, en 2000. De ellos se colige que la protección de la maternidad en el trabajo tiene como objetivo garantizar que las actividades realizadas por las trabajadoras no supongan ningún riesgo para su salud y la de su hijo, y asegurar que su función procreadora no comprometa su seguridad económica y del empleo. La protección de la maternidad para las mujeres trabajadoras constituye un elemento esencial de la igualdad de oportunidades y protección de la salud. Trata de permitir a las mujeres combinar satisfactoriamente sus funciones procreadora y productiva e impedir el trato desigual en el empleo a causa de su función reproductiva. Un objetivo importante consiste en reforzar la protección de la maternidad y lograr ampliarla a todas las mujeres que trabajen en los diversos sectores de la economía (TRAVAIL, 2012). En Convenio Número 183 y la Recomendación Número 191, se destacan los elementos esenciales de la protección de la maternidad en el trabajo:

- Licencia de maternidad: el derecho de las mujeres a un período de descanso en el trabajo en relación con el embarazo, el parto y el posparto.

- Prestaciones pecuniarias y médicas: el derecho de la madre a prestaciones pecuniarias durante su ausencia por maternidad y a la atención médica en relación con el embarazo, el parto y los cuidados posnatales.

- Protección de la salud en el lugar de trabajo: para la madre y el feto durante el embarazo, así como durante la lactancia.

- Protección del empleo y no discriminación: garantía de la trabajadora a la seguridad del empleo y al derecho de reincorporarse tras su licencia de maternidad al mismo trabajo o a uno equivalente con el mismo salario que percibía con anterioridad a la licencia de maternidad. Además, una mujer no puede ser discriminada durante en el trabajo o durante el período de búsqueda del mismo por razones de su función reproductiva.

- Dispositivos para la lactancia: para ayudar a las trabajadoras a amamantar o extraerse leche en el lugar de trabajo durante un período determinado tras la reincorporación al trabajo. 


\section{DERECHO COIMPARADO EN CUANTO A LAS TRHA}

En Argentina ha entrado en vigor en 2015 un nuevo Código Civil y Comercial que introduce como una tercera fuente de filiación: la voluntad de procrear operativa mediante las técnicas de reproducción asistida ${ }^{19}$. La regulación vigente en Argentina se apoya en los principios constitucionales, tales como el derecho a fundar una familia y a la salud sexual y reproductiva, con la pretensión de ampliar derechos, atendiendo a la evolución de la sociedad. La Ley de Acceso integral a las Técnicas de Reproducción Asistida o Ley $26.862 / 2013$ y su Reglamento 953 de $2013^{20}$ se complementa con las reglas del nuevo Código Civil, particularmente los artículos 558 a 593, aplicables a la determinación de la filiación de los niños nacidos por TRHA. Se reconoce el derecho a saber del nacido mediante TRHA con material de tercero. Se establece la obligación del Estado de resguardar toda la información relativa a los donantes, adoptándose una solución intermedia en lo que respecta al levantamiento del anonimato del donante que puede lograrse exponiendo razones fundadas en un proceso judicial. En cuanto a la práctica de la gestación por sustitución no está prohibida, pero tampoco regulada en Argentina, por lo que, aun silenciada por el Código, ha pasado a formar parte de la realidad social, habiendo logrado reconocimiento en algunos casos mediante polémicas sentencias judiciales.

Los informes sociológicos obtenidos en algunos de los países iberoamericanos muestran cierta desinformación acerca las potencialidades y dilemas éticos que plantean los avances científicos en el campo de la genética humana y las prácticas biotecnológicas, favoreciendo así la falta de regulación al predominio de las reglas del mercado. Referencias aisladas a las TRHA como la que hace genéricamente el Código Civil chileno en su artículo 182 para establecer la filiación de los nacidos mediante las mismas, deja en la penumbra muchas cuestiones acerca de la licitud de tales técnicas. También resulta difícil de coordinar las normas del Código Penal para el Distrito Federal de México de 2002 en relación con la práctica de la IA, FIV y manipulación genética, con la legislación sanitaria aplicable en la República Federal Mexicana donde los contratos de gestación subrogada no tienen validez legal, pero tampoco están prohibidos, aunque sí cuentan con regulación protectora de la mujer gestante y del nasciturus en el Estado de Tabasco.

\footnotetext{
${ }^{19}$ Artículo 558: La filiación puede tener lugar por naturaleza, mediante técnicas de reproducción humana asistida o por adopción. La filiación por adopción plena, por naturaleza o por técnicas de reproducción humana asistida matrimonial o extramatrimonial, surten los mismos efectos, conforme a las disposiciones de este Código. Ninguna persona puede tener más de dos vínculos filiales, cualquiera que sea la naturaleza de la filiación.

${ }^{20}$ Vid Ley 26.862-Reproducción médicamente asistida y reglamentación, disponible en www.msal.gob.ar, consultado el 1 de noviembre del 2019
} 
Los principios rectores de la Bioética latinoamericana, los que podrían informar las futuras regulaciones de las TRHA, son la dignidad humana; respeto a los derechos humanos; no comercialización del cuerpo humano; no discriminación; conservación y aprovechamiento sustentable de la diversidad biológica, libre investigación de la paternidad y maternidad genéticas y libertad de investigación científica en un marco de referencias éticas.

\section{REGULACIÓN DE LAS TRHA EN CUBA}

En Cuba ha habido un proceso de adaptación a convenios y legislaciones internacionales, es por ello que las diferentes políticas estatales han buscado integrar a la mujer con plenos derechos en el mercado laboral en virtud del principio protectorio. En consonancia con ello y al ser Cuba miembro de la OIT, varios de los convenios de la organización han sido ratificados por el país, a tono con la normativa constitucional (Artículo 8) ${ }^{21}$, constituyendo una de las fuentes formales del Derecho Laboral Cubano (Viamontes, 2005, p. 38). En materia de maternidad, Cuba ha ratificado estos convenios (NORMLEX).

Este tema tiene una trascendencia para Cuba, manifestándose en la normativa constitucional pues se regula el aseguramiento por parte del Estado de los derechos sexuales y reproductivos de la mujer ${ }^{22}$, el derecho a fundar familia ${ }^{23}$ y la protección por parte del Estado de la maternidad y la paternidad ${ }^{24}$. En el Lineamiento No. 116 de la Política Económica y Social del Partido y la Revolución que atañe al contexto demográfico, en el Decreto-Ley No.339/2017 que amplía y establece nuevos derechos para la madre y el padre, para la familia en general, para la protección de la maternidad y el cuidado de los

${ }^{21}$ Artículo 8 de la Constitución de la República de Cuba (2019), donde se establece: Lo prescrito en los tratados internacionales en vigor para la República de Cuba forma parte o se integra, según corresponda, al ordenamiento jurídico nacional. La Constitución de la República de Cuba prima sobre estos tratados internacionales.

22 Vid. Artículo 43 de la Constitución cubana (2019): La mujer y el hombre tienen iguales derechos y responsabilidades en lo económico, político, cultural, laboral, social, familiar y en cualquier otro ámbito. El Estado garantiza que se ofrezcan a ambos las mismas oportunidades y posibilidades. El Estado propicia el desarrollo integral de las mujeres y su plena participación social. Asegura el ejercicio de sus derechos sexuales y reproductivos, las protege de la violencia de género en cualquiera de sus manifestaciones y espacios, y crea los mecanismos institucionales y legales para ello.

${ }^{23}$ Vid. Artículo 81 de la Constitución cubana (2019): Toda persona tiene derecho a fundar una familia. El Estado reconoce y protege a las familias, cualquiera sea su forma de organización, como célula fundamental de la sociedad y crea las condiciones para garantizar que se favorezca integralmente la consecución de sus fines.

Se constituyen por vínculos jurídicos o de hecho, de naturaleza afectiva, y se basan en la igualdad de derechos, deberes y oportunidades de sus integrantes.

La protección jurídica de los diversos tipos de familias es regulada por la ley

${ }^{24}$ Vid. Artículo 84 de la Constitución cubana (2019): La maternidad y la paternidad son protegidas por el Estado.

Las madres y los padres tienen responsabilidades y funciones esenciales en la educación y formación integral de las nuevas generaciones en los valores morales, éticos y cívicos, en correspondencia con la vida en nuestra sociedad socialista. Las madres y los padres u otros parientes consanguíneos o afines que cumplan funciones de guarda y cuidado tienen el deber de dar alimentos a niñas, niños y adolescentes, respetar y garantizar el pleno ejercicio de sus derechos, protegerlos de todos los tipos de violencia y contribuir activamente al desarrollo pleno de su personalidad. Los hijos, a su vez, están obligados a respetar, atender y proteger a sus madres, padres y otros parientes, conforme con lo establecido en la ley 
hijos menores, propicia una mayor integración de la familia en el cuidado de los niños y estimula la permanencia y reincorporación de la mujer al trabajo. El Decreto-Ley No.340 regula, para la trabajadora que se encuentra en el disfrute de la maternidad, enferma o accidentada, el tiempo de contribución a los efectos de obtener el derecho a la prestación económica y exonera a estas mujeres de la obligación de contribuir a la seguridad social. Por supuesto desde la normativa laboral patria, a saber el Código de Trabajo ${ }^{25}$ y su Reglamento ${ }^{26}$, toda vez que dentro del conjunto de derechos reconocidos se encuentran los derechos de trabajo y de seguridad social que se confieren a la trabajadora, para proteger su maternidad y facilitar su atención médica, el descanso pre y postnatal y el cuidado de los hijos menores ${ }^{27}$.

Sin embargo, esa capacidad de procreación puede verse afectada lo cual pudiera acarrear el sometimiento a procedimientos de reproducción asistida. Para ello se creó en el año 2007, el Programa Nacional de Atención a la Pareja Infértil, que tiene como objetivo brindar cobertura nacional a la atención integral y escalonada en todos los Niveles del Sistema Nacional de Salud a esta problemática en Cuba, el cual ha sido paulatinamente perfeccionado para poder facilitar la atención a las parejas en las consultas municipales, los centros provinciales de reproducción asistida y las instituciones de alta tecnología. El país cuenta con tres niveles de atención de salud a las mismas que discurren desde (Zaldívar, 2017, p. 84):

1. Nivel primario o Consulta de atención a la pareja infértil: se realizan estudios, que incluyen una serie de análisis complementarios tanto al hombre como a la mujer, para la evaluación, diagnóstico y orientación a la pareja. En la actualidad, prácticamente todos los municipios del país cuentan con una consulta de atención a la pareja infértil, ubicada en uno de sus Policlínicos, contando con personal preparado para ello.

2. Nivel secundario o Consulta Multidisciplinaria de Infertilidad a nivel hospitalario: A este nivel se profundizan los estudios para diagnóstico del nivel primario. Se proyecta que en cada Provincia será creado un Centro de Reproducción Asistida de Baja Tecnología (RABT), que estará ubicado en un Hospital General o Gineco-obstétrico.

${ }^{25}$ Vid. Ley No. 116/2013 Código de Trabajo en GO Extraordinaria No. 29 de 17/6/2014.

${ }^{26}$ Vid. Decreto No. 326/2014 Reglamento del Código de Trabajo en GO Extraordinaria No. 29 de 17/-6/2014.

${ }^{27}$ Vid. Artículo 2 inciso i del Código de Trabajo. 
3. Nivel terciario o Servicios de Infertilidad: correspondiente a los Centros de Reproducción de Alta Tecnología (RAAT) de alta tecnología, como el hospital Holguinero Vladimir I. Lenin, Gustavo Aldereguía Lima, en Cienfuegos, y los centros capitalinos Ramón González Coro, América Arias, Hermanos Ameijeiras. Sólo en ese nivel pueden aplicarse soluciones avanzadas como la fertilización in vitro (FIV), la inclusión citoplasmática (ICSI), el abordaje quirúrgico por factor masculino severo y la donación de óvulos (ovodonación). A este nivel se reanaliza cada caso, a las parejas que lo requieran se les realizarán los estudios necesarios para confirmar o completar el diagnóstico y tratamiento (por ejemplo, estudio de posibles causas inmunológicas).

\section{ELEMENTOS FUNDAMENTALES DE SU REGULACIÓN EN CUBA. DESAFÍOS PARA UNA SOCIEDAD HETEROGÉNEA}

Se considera que el vínculo que engendra la filiación genera una serie de efectos que irradian todo el campo del Derecho, desde la determinación de los apellidos, los derechos asociados al status de ciudadano, la obligación de los padres de proveer el adecuado desarrollo de los menores, proporcionar alimentos hasta los derechos sucesorios. Actualmente la maternidad y la paternidad no siempre devienen del hecho biológico de la procreación. Por ello existen padres y madres también por adopción o por utilización de TRHA. Ahora, respecto a la voluntad de determinar quién sea el padre; en las TRHA se sustituye el factor biológico por el volitivo, eligiendo la fuente del material genético determinado por el usuario. Como resultado de esa voluntad proceacional se constituyen hoy diferentes tipos de familias: familias homoparentales constituidas por dos padres o por dos madres; familias monoparentales, familias heterosexuales. $Y$ es esta cosmopolita sociedad lo que impone nuevos retos a la comunidad jurídica, pues todos son seres humanos y les ampara el derecho de fundar una familia. Incluso traspasa las fronteras de las relaciones jurídicas familiares para encontrar su fundamento iusfilosófico en la dignidad del ser humano para determinados tipos de familias. A partir de aquí se provocan problemas en cuanto a la determinación de los vínculos paterno-filiares y de todos los efectos jurídicos que ello provoca. Estos efectos también se trasladan, como se ha explicado, a la esfera laboral. Al revisar la normativa laboral ${ }^{28}$ y la Ley de la Maternidad cubana ${ }^{29}$, es posible percatarse que existe una protección a la trabajadora en lo concerniente a la maternidad, estas normas jurídicas cumplen con

\footnotetext{
${ }^{28}$ Vid. Capítulo IV del Código de Trabajo (artículos 59-63) y Reglamento del CT, Decreto No.326/2014 (artículos 84-85)

${ }^{29}$ Vid. Artículo 6 del Decreto Ley No. 339/2017 que establece que se entiende por:

a) Licencia por maternidad pre y posnatal: es la suspensión de la relación de trabajo de la madre con carácter obligatorio, para garantizar su descanso ante la proximidad del parto, así como para su recuperación posterior y la atención del menor; es retribuida o no, en correspondencia con el cumplimiento de los requisitos establecidos en este Decreto Ley; comprende las seis (6) semanas anteriores al parto y las doce (12) posteriores a este;
} 
los elementos esenciales de la protección de la maternidad en el trabajo: el empleador debe crear y mantener condiciones de trabajo para la mujer; la trabajadora gestante que por prescripción médica no puede permanecer en el cargo por considerarse perjudicial al embarazo, recibe la protección que establece la legislación de la maternidad; la trabajadora gestante, cualquiera que sea la actividad que realiza, está en la obligación de recesar en sus labores en los términos y condiciones que establece la legislación específica; durante el embarazo y posterior al parto, la trabajadora tiene derecho a disfrutar de licencia retribuida en la forma y cuantía establecidas respecto a la maternidad de la trabajadora para el régimen general o los especiales de seguridad social.

Sin embargo, en las normativas nada se refiere sobre la protección a las trabajadoras que no pueden tener hijos espontáneamente y deben recurrir a los procedimientos requeridos para lograr este fin ${ }^{30}$, y que deben ausentarse por estas razones de su centro laboral, y como toda problemática social a la que el Estado dedica especial atención, la implementación de las Técnicas de Reproducción Asistida en Cuba requiere de un mecanismo de tratamiento legislativo e institucional, que lo organice y haga efectivo.

En virtud de la utilización de las TRHA debemos ampliar la concepción tradicional sobre la maternidad y la filiación con el objetivo de proteger los derechos reproductivos y sexuales garantizando el libre desarrollo de la personalidad humana. Por ello se debe reconocer y respetar la voluntad de cada ser humano de procrear mediando o no las TRHA. No solo como alternativa a la infertilidad o la esterilidad sino también producto a condicionamientos personales y sociales como: el desarrollo profesional, la decisión de fundar familias monoparentales y homoparentales, enfermedades de transmisión sexual, cáncer, entre otras.

En el ámbito laboral no se debe obviar la finalidad tuitiva y protectora de esta rama del Derecho como principio general que lo informa. Por tanto, la normativa debe interpretarse en plena correspondencia con este principio y los derechos fundamentales reconocidos pues la Constitución es fuente formal del

\footnotetext{
b) prestación económica: es la cuantía que percibe la madre durante el período de licencia retribuida por maternidad pre y posnatal;

c) prestación social: es la cuantía que se otorga a la madre, padre o familiar a quien se encargue el cuidado del menor al vencimiento de la licencia postnatal y hasta que este arribe a su primer año de vida. Las cuantías de estas prestaciones mensuales no pueden ser inferiores al salario mínimo vigente en el país; de ser así, se elevan hasta dicha cuantía.

${ }^{30}$ No existe una disposición normativa con rango de Ley que se haya dedicado a la materia en cuestión. Existe una resolución del Ministerio de Salud Pública, la Nº61/2014, que regula el procedimiento excepcional para la aplicación de la ovodonación, reconociendo a la misma como una técnica de reproducción asistida de alta complejidad. La misma, establece una definición del procedimiento en la que incluye la calificación del tratamiento como una técnica excepcionalísima, donde la donante para el procedimiento puede ser familiar o anónima y la paciente receptora debe estar integrada al servicio de Reproducción Asistida del Sistema Nacional de Salud.
} 
Derecho del Trabajo. En el caso de la Constitución cubana se evidencia un reflejo de los derechos reproductivos y sexuales y la obligación estatal de garantizarlos. Por ello se deben crear mecanismos jurídicos-laborales que evidencien como se reconoce, se promueve, garantiza y respeta los preceptos constitucionales. Debe ser protegida la mujer trabajadora en el proceso de preconcepción sobre la base de la reproducción artificial ya que en virtud de la regulación existente no hay manifestación efectiva.

Una alternativa podría ser extender el alcance de protección de la Ley de Maternidad con la que contamos en aras de ofrecer resguardo a los derechos laborales ya reconocidos durante el proceso de aplicación de las TRHA. Nos referimos específicamente a percibir una remuneración económica durante el tiempo en que encuentra sometida a estos procederes médicos, a exceptuarse por motivos justificados a la jornada laboral sin afectaciones del salario ni que conlleven a una culminación de su contrato de trabajo que lesionen el derecho al trabajo como derecho constitucional. Ello posibilita que se cumpla con los indicadores que garanticen el empleo digno, no solo desde normativas jurídicas, sino desde políticas publicas eficientes en la materia que garanticen el correcto funcionamiento de instituciones y la concientización de empleadores.

También se puede ofrecer una reinterpretación de las licencias retribuidas por maternidad (específicamente las pre natales), y sustentando esta posibilidad, no en el hecho de encontrarse ya la trabajadora en el período gestacional, sino en la intención de materializar ese deseo. Debe, por tanto, la licencia anticiparse al embarazo y otorgarse cuando la persona informa a su empleador que se someterá de manera individual o junto a su pareja si la tiene a un tratamiento de infertilidad. También deben involucrarse valores tales como la solidaridad y la comprensión hacia la trabajadora o el trabajador pues estos procesos tienen costes físicos, emocionales y económicos como la decepción, la incertidumbre, la depresión ante no lograr el objetivo; todo lo cual puede provocar un bajo rendimiento en cuanto a las obligaciones laborales, de ahí que sea tan importante el pensar en una licencia preconcepción.

Resulta fundamental asimilar los cambios que se están produciendo en la percepción social de los roles de los progenitores, así como los estereotipos predominantes. Por ello debemos hablar de las licencias de paternidad pues las medidas de conciliación de la vida laboral y familiar interesan tanto a los hombres como a las mujeres. Si bien en nuestro país se hace alusión a las licencias no retribuidas a los padres, no se refiere tampoco al período preconcepción mediante la reproducción artificial. 
Ante todo, se deben prevenir y eliminar la discriminación contra las mujeres y los hombres con responsabilidades familiares derivadas de la necesidad personal de concebir artificialmente, y protegerlos en su relación de trabajo ante las arbitrariedades que puedan generarse. La falta de garantías formales de estos derechos podría provocar situaciones de indefensión hacia los trabajadores de cara a los poderes de dirección y control del empleador. Al no establecerse en ley y ante la necesidad práctica de ausentarse, llegar tarde, requerir tratamiento durante el proceso de utilización de estas técnicas pueden provocarse situaciones injustas que se consideran legales. Se considera primordial crear una cultura favorable en el lugar de trabajo y que los procesos de utilización de las técnicas de reproducción humana asistida se inserten en la dinámica laboral poniendo a disposición de los trabajadores opciones de conciliación de su empleo con el deseo de ser padres o madres. Sobre todo, que estas opciones se positiven y se conviertan entonces en garantías de los derechos de los trabajadores, que funcionen como armas de defensa.

\section{CONCLUSIONES}

Los avances científicos influyen en gran medida en las relaciones jurídicas laborales por lo que se hace necesario la adecuación de los preceptos legales a los nuevos cambios que impone la Ciencia, la Medicina y la Tecnología. Las TRHA son una evidencia fehaciente de esta realidad. Resulta evidente a partir de la normativa internacional relacionada con la TRHA que se hace necesario las limitaciones a la reproducción artificial en aras de evitar la comercialización de material genético con fines lucrativos. Además, se debe adecuar la concepción que existe sobre la maternidad en Cuba a la alternativa de procreación con la que contamos por medio de las TRHA, en aras de proteger la salud reproductiva y el desarrollo integral de los trabajadores.

En el ámbito laboral se considera inminente la correcta regulación jurídica de estos procederes para evitar actuaciones arbitrarias del empleador que lesionen los derechos laborales y reproductivos de los trabajadores; ofrecer alternativas de licencias, no afectar económicamente al trabajador durante el proceso de procreación artificial. En Cuba a pesar de existir programas y políticas públicas en aras de proteger a la pareja infértil no contamos con una normativa jurídica laboral que protejan estas prácticas de preconcepción lo cual desampara al trabajador ubicándolo en un estado de indefensión.

\section{BIBLIOGRAFÍA}

- ARÁMBUlA REYeS, A (2008). Maternidad subrogada. México: Ediciones B. 
CLABORJURI5

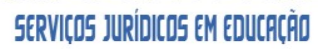

REVISTA DE DIREITO DO TRABALHO, PROCESSO DO TRABALHO E DIREITO DA SEGURIDADE SOCIAL
Julho - Dezembro

v.4, n.2, 2020

ISSN: 2674-6913

- Campos Vargas, G.

(2012)

Implicaciones

éticas,

legales,

religiosas

$\mathrm{y}$ socioeconómicas de la fecundación in vitro en la Salud Reproductiva de Costa Rica. Revista Enfermería Actual en Costa Rica, disponible en: < http://www.revenf.ucr.ac.cr/vitro.pdf> (consultado el 16 de octubre de 2019)

- COLECTIVO DE AUTORES (2017). El acceso a las técnicas de reproducción asistida como una garantía de los Derechos Sexuales y Reproductivos: la jurisprudencia de la Corte Constitucional a la luz del Derecho Internacional de los Derechos Humanos. Revista Justicia No. 31, enero-junio.

- CoOK, R. J; Dickens, B. M y Fathalda, Mahmoud F (2003) Salud reproductiva y derechos humanos: integración de la medicina, la ética y el derecho.

- Del CaRmen VALDÉS DíAZ, C. (2017) El acceso a algunas técnicas de reproducción humana asistida: “Crónica de una vida anunciada”. México: Revista del Instituto de Ciencias Jurídicas de Puebla No.39 enero-junio.

- DuRAnd CARrión, J. (2009) Las Técnicas de Reproducción Asistida y su Regulación, Un reto para el Derecho, al Ciencia y la tecnología. San Martin, Tesis destinada a obtener el Doctorado en Derecho por la Universidad Particular de SanMartin de Porres, disponible en:<https://riunet.upv.es/bitmigracion.ucm.es/info/medlegal/5\%20Escuelastida.pdf?s $>$ (consultad o el 16 de octubre de 2019)

- GIL, M. (2016) Reproducción asistida: antecedentes y legislación. La ley que amplía derechos de toda persona a la paternidad o maternidad (2016), Revista digital UNIDIVERSIDAD (14 de mayo)

- Glosario de Terminología en Técnicas de Reproducción Assistida (TRA).Versión revisada y preparada por el International Committee for Monitoring Assisted

- La Familia monoparental, disponible en:<http://dialnet.unirioja.es> (consultado el 16 de octubre del 2019)

- Reproductive Technology (ICMART) y la Organización Mundial de la Salud (OMS) Traducido y Publicado por la Red Latinoamericana de Reproducción Asistida en 2010 @ Organización Mundial de la Salud 2010 en:<https://www.who.int/reproductivehealth/publications/infertility/art_terminology2/es/>.

- PÉREz Fuentes, G. (1997). Algunas reflexiones jurídicas sobre el tema de la esterilidad y las técnicas de reproducción asistida. Cuadernos de Bioética

- Pérez González, A. (2016) Homoparentalidad, un nuevo tipo de familia. Santiago de Chile: Tesis para optar al grado de Licenciado en Ciencias Jurídicas y Sociales.

- Sociedad Española de Fertilidad (SEF). Saber más sobre Fertilidad y Reproducción asistida, Madrid, disponible en:<www.sefertilidad.com>, consultado el 15 de octubre del 2019. 
- TURNER SAELZER. (2001) Los derechos sexuales y reproductivos y su incidencia en la esterilización y la procreación asistida, Revista de Derecho, Vol. XII, No.2.

- Viamontes GullBeAux, E. (2005). Derecho Laboral Cubano, Teoría y Legislación, Tomo I. La Habana: Editorial Félix Varela.

- ZaLdívar Marrón, S. (2017) Presupuestos iusfilosóficos para la regulación de las técnicas de reproducción asistida en Cuba. La Habana: Tesis presentada en opción al título de Licenciado en Derecho; Facultad de Derecho de la Universidad de La Habana.

\section{Normas Jurídicas}

- Constitución de la República de Cuba, publicada en Gaceta Oficial Extraordinaria No. 5 de 2019.

- Código Civil y Comercial de Argentina

- Ley No. 116/2013 Código de Trabajo publicado en Gaceta Oficial Extraordinaria No. 29 de 17 de junio de 2014.

- Decreto 326, Reglamento del Código de Trabajo GO Ext No.29 17/06/2014

- Argentina, Ley 26.862-Reproducción médicamente asistida y reglamentación, disponible en: <www.msal.gob.ar>, consultado el 1 de noviembre del 2019

- Decreto No. 326 Reglamento de la Ley. No 116/2013 Código de Trabajo publicado en Gaceta Oficial Extraordinaria No. 29 de 17 de junio de 2014.

- Decreto Ley No. 339 "De la maternidad de la trabajadora", publicado en Gaceta Oficial Extraordinaria No. 7 de 10 de febrero de 2017.

- Convenio 3 de la OIT sobre la libertad sindical y la protección del derecho de sindicación, 1919.

- Convenio 103 de la OIT sobre el derecho de sindicación y de negociación colectiva, 1952.

- Convenio 183 de la OIT sobre la libertad sindical y la protección del derecho de sindicación, 2000.

- Convenio de Oviedo-Bioética, disponible en: <www.bioeticanet.info>, consultado el 1 de noviembre del 2019.

\section{Otros Documentos}

- Lineamientos de la Política Económica y Social del Partido y la Revolución para el Período 2016-2021.

- Declaración Universal sobre el Genoma Humano y los Derechos Humanos, disponible en: <http://portal.unesco.org>, consultado el 1 de noviembre del 2019.

- “Declaración de Bioética de Gijón”, disponible en: <http://www.sibi.org/es/12-declaraciones/68declaracion-bioetica-de-gijon.html>, consultado el 1 de noviembre del 2019.

- Declaración de las Naciones Unidas sobre la Clonación Humana", disponible en <http://wwwbioeticaweb.com>, consultado el 1 de noviembre del 2019. 
- "Declaración Universal sobre Bioética y Derechos Humanos" disponible en: <http://portal.unesco.org/es/ev.phpURL_ID=13178\&URL_DO=DO_TOPIC\&URL_SECTION=201.htm l>, consultado el 1 de noviembre del 2019. 